\title{
Multiple sensor outputs and computational intelligence towards estimating state and speed for control of lower limb prostheses
}

\author{
Pamela A. Hardaker, Benjamin N. Passow and David Elizondo \\ De Montfort University's Interdisciplinary Group in Intelligent Transport Systems (DIGITS) \& Centre \\ for Computational Intelligence (CCI), De Montfort University, Leicester, LE1 9BH, United Kingdom \\ pamela.hardaker@ieee.org, benpassow@ieee.org, elizondo@dmu.ac.uk
}

\begin{abstract}
For as long as people have been able to survive limb threatening injuries prostheses have been created. Modern lower limb prostheses are primarily controlled by adjusting the amount of damping in the knee to bend in a suitable manner for walking and running. Often the choice of walking state or running state has to be controlled manually by pressing a button.

This paper examines how this control could be improved using sensors attached tofa the limbs of two volunteers. The signals from the sensors had features extracted which were passed through a computational intelligence system. The system was used to determine whether the volunteer was walking or running and their movement speed.

Two new features are presented which identify the movement states of standing, walking and running and the movement speed of the volunteer. The results suggest that the control of the prosthetic limb could be improved.
\end{abstract}

\section{INTRODUCTION}

The development of effective and functional prostheses has become increasingly important as more people survive limb threatening injuries. In [1] we learn that there are 32 million amputees worldwide and that over $75 \%$ of major amputations are lower limbs.

While many of the problems facing those trying to produce functionally realistic prostheses are being solved the way in which the units react to changes in the movement state and speed of the wearer still has to be controlled manually.

This paper examines whether this area could be improved by using features extracted from sensors placed on the prosthesis and on the prosthesis wearer's body. Such features include the average sensor output calculated over a sliding window and rate of change of a sensor output over two samples. A computational intelligence (CI) system was used to determine from the features whether the volunteer was walking or running and what speed they were moving at. This work continues the work described in [2].

Recent improvements in all areas, including materials, computer processing times and battery technology, mean that a microprocessor controlled prosthesis is now a reality. However, the challenge has always been to create a system that could be wearable and usable. The following literature review looks at current research in these areas. This paper is divided into seven sections. A literature review is presented in section two, feature extraction is discussed in section three and pattern classification in section four. In section five the experimental setup is outlined. The results are presented in section six and final conclusions are drawn in section seven.

\section{LiterATURE REVIEW}

In general, there are two ways in which computational intelligence can be used for prostheses control. The first is interpreting signals from the wearer to determine what movement they are making and the second is in controlling the actions of the limb, here there is a large overlap between the development of prostheses and that of robots and exoskeletons.

In all cases the artificial limbs must move and balance like a human limb to be successful. Again, this area of control can be split into two. The first being simple pattern recognition to determine a state for the prosthesis from the incoming signals. The second is in the control of the entire walking gait of a bipedal robot.

\section{A. The use of sensors in prosthetic limb control}

Several types of sensor have been used to help with the control of a prosthetic limb. The current state of the art in the use of these sensors is considered in the following subsections.

1) Electromyographic sensors: Figure 1 shows a typical EMG sensor output with examples of the signal recorded during walking and running.

Much research has been done into the fact that the residual limb can still give the signals required to control the missing portion of the limb and in [3] the challenges of doing this are discussed. One of the main challenges is the fact that each step from each individual will produce different signals at different times making pattern recognition very difficult. In [4] the fact that the signals that reach the surface mounted EMG sensor have had to pass through other muscles, tissue and bone, all of which will have an effect upon the signal is discussed. 


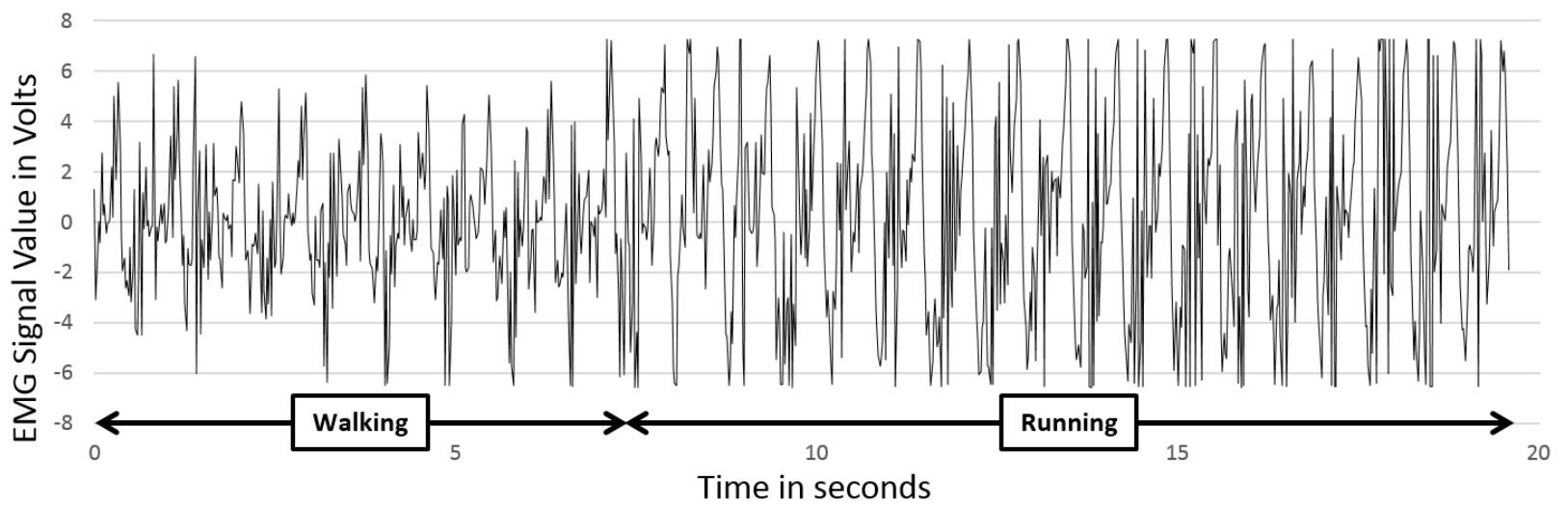

Fig. 1: EMG sensor output taken from a volunteer's thigh while walking and running.

The use of signals from an EMG sensor to control a prosthetic arm were being reported on as early as 1967 [5] to simply open and close a hand. Now the research has developed to the point where EMG signals are being used in real time to control a prosthetic hand [6]. In [3] an EMG sensor is used to distinguish seven movement modes for a lower limb prosthesis including level-ground walking and turning. In this work three movement states are distinguished, standing still, walking and running.

Volitional control of the muscles has been examined by [7] where the quadriceps and hamstring muscle EMG signals were used to directly interpret the users intent to flex or extend the knee.

It is very easy to think only of adapting the prosthetic to fit the person. However, in some circumstances surgery has been performed to transfer nerves to a more accessible position for the EMG sensors. This has then allowed signals to be detected that would otherwise have been impossible to find. This has been used in a number of situations for upper limb prosthesis [3, 8].

It is suggested that this technique could be used for lower limb amputees in the future. In [9] the nerves that used to control the part of the limb that has been amputated were transferred to a spare muscle then surgically re-innervated. This technique allows the signal that would have been sent to the missing part of the limb to be more effectively captured and used to control a prosthetic.

In $[10,11]$ the authors used EMG signals to directly control an exoskeleton. This has potential for assisting disabled people who are no longer able to walk and for rehabilitating someone with a disability. EMG also has extensive application for analysing the walking gait of non-amputee's [12] and this research could also help in the development of a successful control system.

2) Pressure Sensors: The walking gait of humans has been very thoroughly studied and from [13] we learn that a typical gait cycle for level ground walking consists of two phases: stance and swing. The stance phase begins at heel strike and terminates upon toe off; the swing phase takes up the remainder of the cycle. In $[14,15]$ and many other papers the usefulness of the pressure sensor for detecting movements within the gait cycle of a prosthetic is examined.

Some studies place the pressure sensor between the residual limb and the socket of the prosthesis. This will give a basic idea of whether or not the prosthetic is currently in a load or non-load bearing state. In [16] the effectiveness of this strategy is compared with the use of EMG sensors in the socket of the prosthesis. Furthermore, some authors have used pressure sensors to determine if the user is sitting or standing.

3) Accelerometers: Accelerometers have been used for a variety of tasks in the evaluation of the movement of limbs. In [17] an accelerometer is used to record and confirm the movement of the user while wearing an EMG sensor. This information is then used to determine any adverse effects the movement of the wearer has on the EMG sensor.

\section{B. Signal processing}

Once signals have been collected from the various sensors they need to be processed to make them usable by a classification system.

1) Pre-processing: In [18] the signal processing consists of passing the raw signal from the pre-amplifier through a low-pass filter. The signal is then rectified by taking the absolute value and normalised using the maximum muscle contraction measured before experimentation. This technique uses a sliding window to calculate the average of the last $\mathrm{n}$ signals in the window.

2) Feature Extraction: The information that comes from the EMG sensor, shown in Figure 1, is in the form of a signal which an ANN would not easily be able to recognise. It is necessary to extract the relevant features that distinguish one state from another. In [19] this is described as the main kernel of classification systems and is essential to the motion command identification. The authors also highlight that it is difficult for a single 


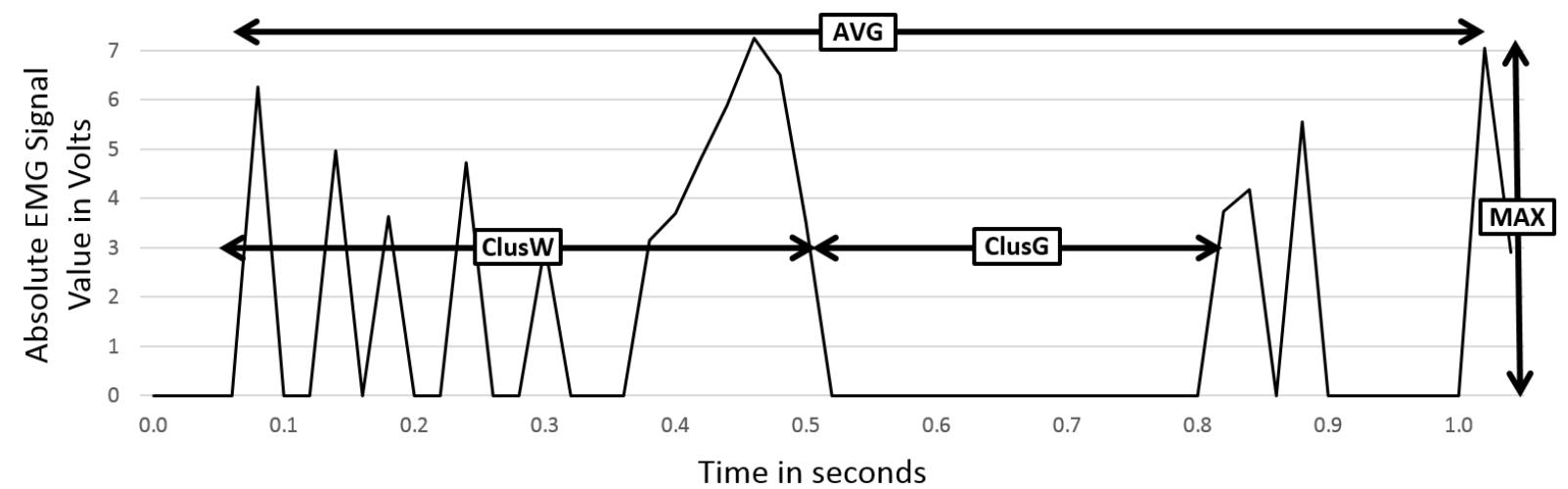

Fig. 2: A visual interpretation of the features extracted from the EMG output.

(Note that the absolute value of the output has been used to allow

the background signal of \pm 2.8 volts to be removed by thresholding)

MAX = maximum of last $\mathrm{n}$ samples $\quad$ ClusW $=$ width of last cluster of spikes

AVG $=$ average of last $\mathrm{n}$ samples

ClusG = gap between last two clusters of spikes

feature to reflect the overall state of the signal. Thus, several different features are required.

In [1] four time-domain features were extracted from the EMG signal, the mean absolute value and the number of zero-crossings, the waveform length, and the number of slope sign changes. These were then used for real-time prediction of the intent to sit or stand.

Other techniques used to extract features include filtering [4]. Most papers agree that the extraction of data from this signal in a timely fashion is one of the hardest aspects of the process.

\section{Pattern classification}

Once the features have been extracted from the EMG signal they need to be used to distinguish between different states. This process involves looking at the patterns within the signal features and then training a classifier to recognise and separate these patterns. Computational Intelligence techniques are often used for this and could include fuzzy mapping functions [19] or different varieties back propagation ANN $[4,20]$.

\section{Controlling a limb or prosthesis}

The final part of the process is to control the limb. In [21] the inputs from the EMG are passed through a dynamic recurrent neural network (DRNN) to control all three sections of a virtual limb on a computer based simulation. A real control system is discussed in [22] where the authors have studied the intricacies of walking at length. They use a feed forward neural network to overcome the limitations of rule based control systems which are unable to take account of changing demands and terrain. A simulation of a powered prosthesis was successfully controlled.

The Plymouth Hand $[4,20,23]$ project has extensively researched the use of an EMG signal to control a prosthetic hand. The authors describe how a single EMG signal is gathered and passed through a series of filters to remove noise from the mains electricity. The signals where then fed into a neural network which had been trained to recognise a series of positions for the hand to move into.

\section{FEATURE EXTRACTION AND PATTERN CLASSIFICATION}

In the previous work [2], several features were extracted from an EMG sensor output. These features, shown in Figure 2, allowed an artificial neural network to distinguish between walking and running by returning a state of 1 for walking or 2 for running.

In the current work extra sensors were added attached via Arduino boards to record new data. These sensors included:

- A wheel sensor attached to a treadmill to record the speed of the volunteer

- Six pressure sensors, one under the heel, toe and ball of each foot

- Four accelerometers, one on the thigh and calf of each leg

The pressure sensor and accelerometer sensors were mounted on a harness so that the volunteer could safely wear them while walking and running on a treadmill as shown in Figure 5. The data was then collected from the sensors as outlined in the following sections.

\section{A. Wheel sensor}

The wheel sensor was attached to the treadmill. The Arduino code for this sensor was set to continuously count the number of times the wheel made a complete revolution. An interrupt was set to trigger every $1 / 50$ of a second and when this interrupt occurred the wheel counter reading was taken and then the counter was zeroed. This gave the number of revolutions made in the last $1 / 50$ of a second and from this the speed of the treadmill and therefore of the volunteer could be 


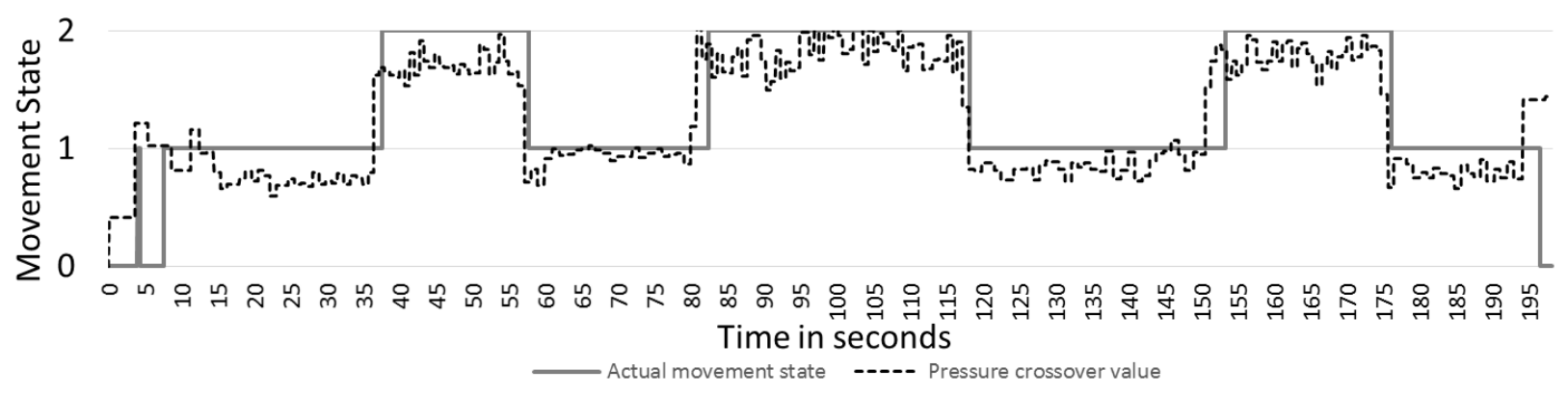

Fig. 3: A comparison of the crossover points of the heel and toe pressures with the actual movement state determined from the wheel speed sensor

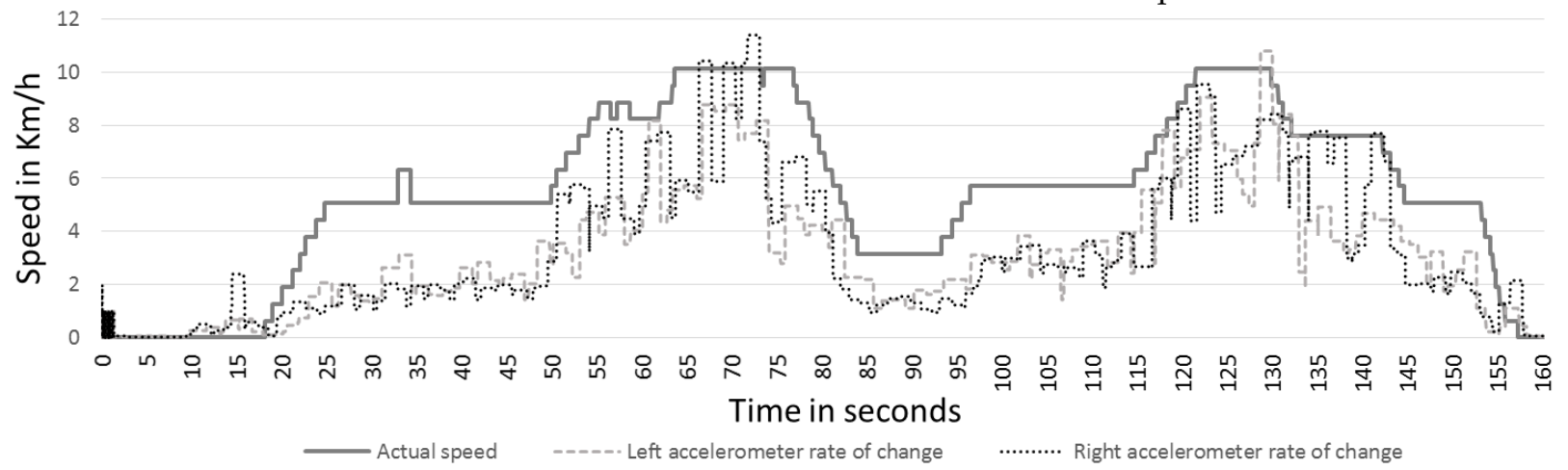

Fig. 4: A comparison of the left and right accelerometer rate of change to the actual speed determined from the wheel speed sensor

calculated in $\mathrm{Km} / \mathrm{h}$. As the wheel sensor experienced some interference, the value achieved was averaged over the last $\mathrm{n}$ readings and $\mathrm{n}$ was varied through experimentation to enhance the results.

\section{B. EMG sensor}

The features derived from the EMG sensor in the previous work were trialled again as part of this work.

\section{Pressure sensors}

The different parts of the walking and running cycle were analysed. From this analysis and examination of the outputs from the pressure sensors, it was found that there was a close correlation between the relative change in heel and toe pressures and whether the volunteer was standing, walking or running.

Figure 3 shows the results of this feature as a black dotted line with the actual recorded movement states of standing (0), walking (1) and running (2) shown in solid grey. As can be seen, there is a strong correlation. However, there is a certain amount of noise at the changeover points. This is because the current method of determining that the recorded movement has gone from walking to running is to threshold it at a given speed and this is clearly open to interpretation. Not only is it possible to walk and run at the same speeds but there is a changeover step where the movement state is half running and half walking as the changeover occurs.
This applies when both accelerating and decelerating. It is also possible that this changeover step is being exaggerated by the delay as the treadmill changes speed.

\section{Accelerometers}

From examination of the accelerometer readings and experimentation a feature was found that was able to approximately estimate the speed at which the volunteer was moving. This feature was found by taking the current accelerometer reading from the previous one to give a rate of change and then averaging this reading over the last $n$ readings, where $n$ was varied through experimentation to enhance the result.

Figure 4 shows the values for this feature for both the left and right accelerometer compared with the speed measured by the wheel sensor (the speed has been normalised for comparison purposes). The two dotted lines show the left and right accelerometer rates of change and as can be seen the general shape of the change correlates well to the measured speed shown in grey.

\section{E. Artificial neural networks}

The new features discovered from the new sensors and the successful features from the previous work were then passed through a series of artificial neural networks for pattern classification. Two different artificial neural network implementations were trialled. The first was the original Multi-Layer Perceptron (MLP) from the Matlab 


\begin{tabular}{|c|c|c|c|c|c|}
\hline Set No. & \multicolumn{5}{|c|}{ Features used as inputs to the ANN } \\
\hline Set $\mathbf{1}$ & ClusG & ClusW & AVG & MAX & \\
\hline Set $\mathbf{2}$ & ClusG & ClusW & AVG & MAX & PRESS \\
\hline Set $\mathbf{3}$ & ClusG & AVG & PRESS & ACCL & ACCR \\
\hline
\end{tabular}

TABLE I: The three sets of features trialled as the inputs to the ANN

Set 1 - original feature set from previous work

Set 2 - original features and new pressure feature

Set 3 - two original features and three new features

artificial neural network toolbox used in the previous work using a tansig transfer function. The second was implemented using the Netlab toolbox [24] which allows a vector of options to tune parameters such as the response function and the gradient optimisation. This was also an MLP with linear output node response and a scaled conjugate gradient (scg) optimisation method. Other response functions and optimisation methods were trialled without success.

Each artificial neural network configuration was trialled with a number of training and testing data sets and the results were compared. As part of the trials, different sets of features were chosen. As can be seen in Table I three feature sets were used. It was found that feature sets 2 and 3 were the most successful and these were taken forward to the main testing.

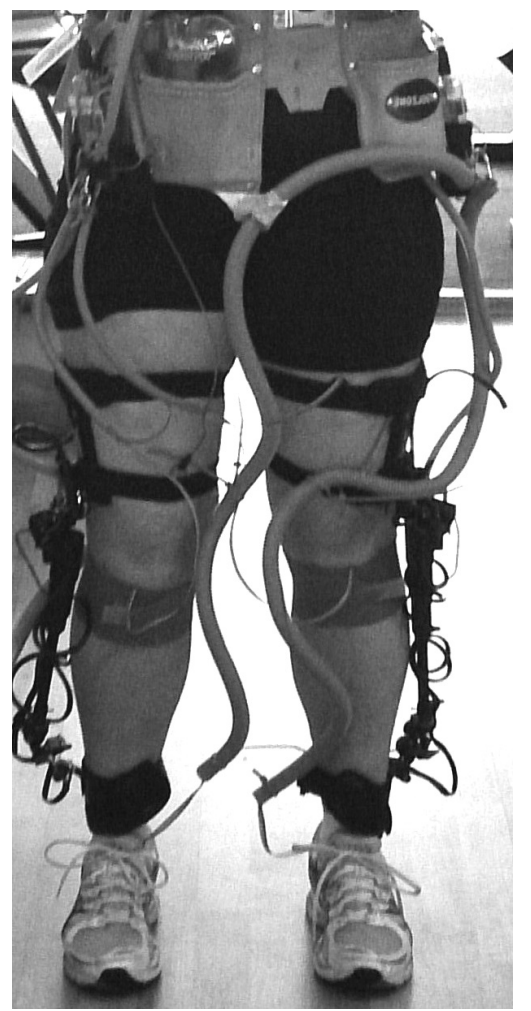

Fig. 5: A volunteer wearing the sensor harness.

\section{EXPERIMENTAL SETUP}

The new sensors were combined with the EMG sensor using Matlab code to collect and process the data.

\section{A. Volunteers}

Data was captured from two volunteers.

- Volunteer one is a 48 year old female with no amputation and is shown in Figure 5.

- Volunteer two is a 30 year old male with one prosthetic leg. At the time of the experiments volunteer two was unable to run on a treadmill and so only walking data could be captured for him.

\section{B. Sensors}

Three Arduino boards were used to interface between the new sensors and the computer, the following sections explain the use of each sensor.

1) Wheel sensor: The wheel sensor was attached to a small, dedicated Arduino board and consisted of a small rubber wheel attached to a rotary encoder which registered a tick each time the wheel completed one rotation. Code was written to send an interrupt to the Arduino board every $1 / 50$ of a second. The ticks were counted until the interrupt occurred at which point the counter was zeroed and the number of ticks were transmitted over a serial connection.

2) Electromyographic Sensor: An EMG sensor was used to pick up the signals sent by the nerves through the leg to control the muscles. The EMG sensor used for this research consists of:

- Motion Lab Systems MA 317 A300 A3 Preamplifier

- Amplifier circuit to further increase signal levels

- Data Translation DT9801 Multifunction USB Data Acquisition Module

- Data Translation QuickDAQ 2014 software

In order to get a strong, accurate output, the placement of the EMG sensor on the volunteer is crucial and needs to be tested before experiments are run. On volunteer two it was found that placing the sensor in the top of the socket was very effective and after positioning the sensor to achieve a good signal, the socket held the sensor in place perfectly throughout the tests.

3) Pressure Sensors: The pressure sensors were attached to the base of the insoles of a pair of trainers. The three sensors were positioned so that they would be under the toe, ball and heel of the foot.

4) Accelerometers: Four accelerometers were used for the experiments these were placed on the left and right thigh and calf. Each accelerometer returned three values giving the amount of movement in the $\mathrm{x}, \mathrm{y}$ and $\mathrm{z}$ planes.

\section{Using the sensors with a treadmill}

The wheel sensor was positioned in such a way that the wheel was held against the treadmill gently but firmly. The two Arduino boards and the Data Translation unit for the EMG sensor were mounted in a wearable tool belt as shown in Figure 5. 


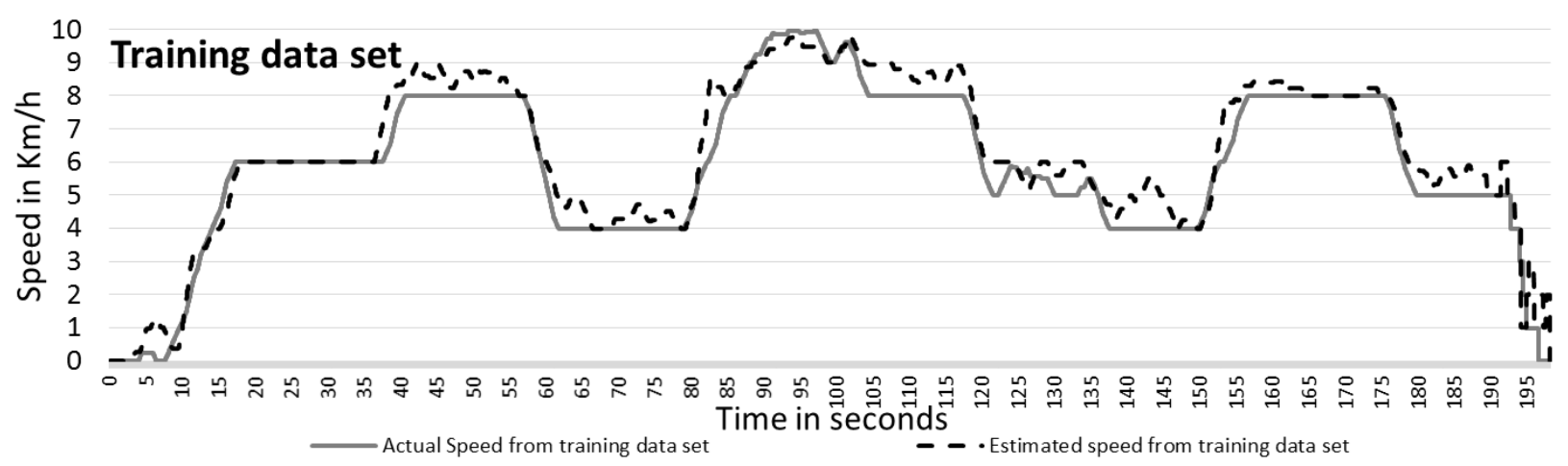

Fig. 6: Example of successful estimation of speed from the training data set.

The actual recorded speed is shown in solid grey and the speed estimated from the features by the artificial neural network in dotted black.

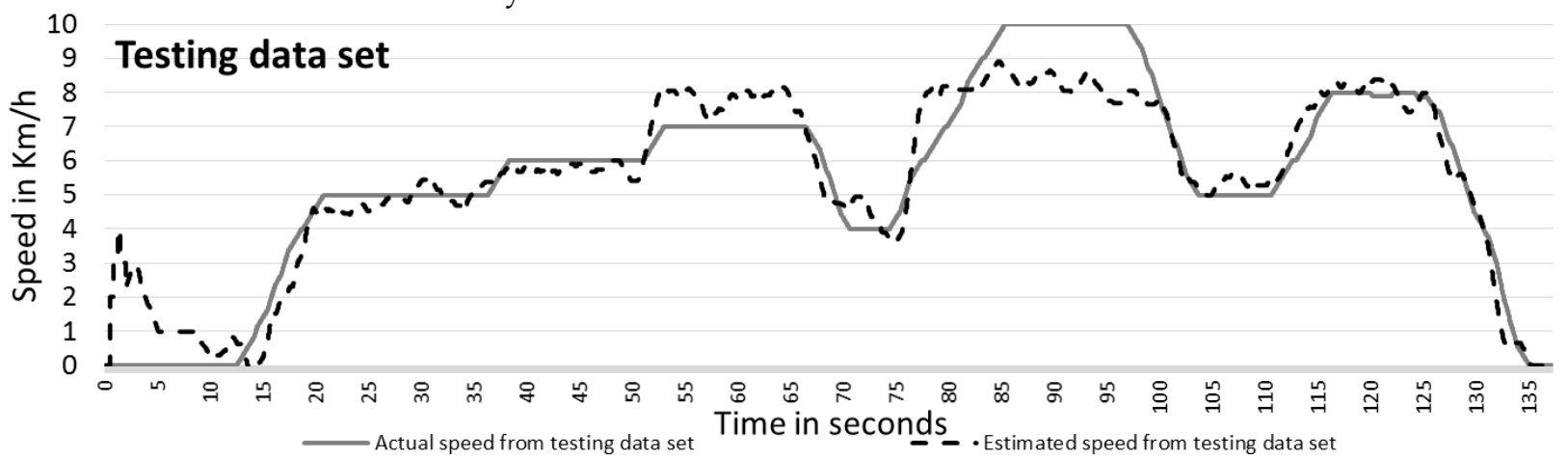

Fig. 7: Example of successful estimation of speed from the testing data set.

The actual recorded speed is shown in solid grey and the speed estimated from the features by the artificial neural network in dotted black.

\begin{tabular}{|c|c|c|c|}
\hline Error type & Feature Set & MSE & RMSE \\
\hline \hline Best - speed & 3 & 1.132 & 1.064 \\
\hline Worst - speed & 2 & 12.398 & 3.521 \\
\hline Best - state & 3 & 0.057 & 0.239 \\
\hline Worst - state & 2 & 0.392 & 0.626 \\
\hline
\end{tabular}

TABLE II: The relative error values from the best and worst runs of the test data for speed and state estimation

\section{Data collection}

Code was written for each Arduino board which implemented a timer interrupt every $1 / 50$ of a second. Each time the interrupt occurred the values of the relevant pins on the Arduino board were written over the serial connection and ExtraPutty was used to capture this. Using this method, four good data sets were acquired.

\section{RESUlts}

The object of this work was to use the features extracted from the new sensors to identify the movement state and movement speed of a volunteer.

\section{A. A meaningful error value}

Trials were carried out using the data sets as both the train and test input in every possible combination. Two outputs were tested, movement state and actual speed. To compare the results the calculated errors from each run of the artificial neural networks were exported to a file along with the relevant settings used for that run and then compared. It was found that no single data set produced better results. This showed that the results were independent of the data set. It was also found that there were no noticeable differences between the results for the different artificial neural network configurations (ie topology and learning parameters).

The best and worst errors found when estimating the actual speed and when estimating the movement state from the test data is shown in Table II. In each case the feature set that was used is shown. As can be seen, feature set 3 produced the best results showing that the new features gave the best estimation.

The MSE and RMSE values shown in this table do not appear promising. However, this form of classification does not require a perfect output. For both movement state and actual speed estimation it is sufficient to be close enough to the required output that rounding will correct the value as will be shown in the next section.

1) Lowest error when estimating speed: Figures 6 and 7 show the results with the lowest error for the training run and testing run respectively when estimating the 


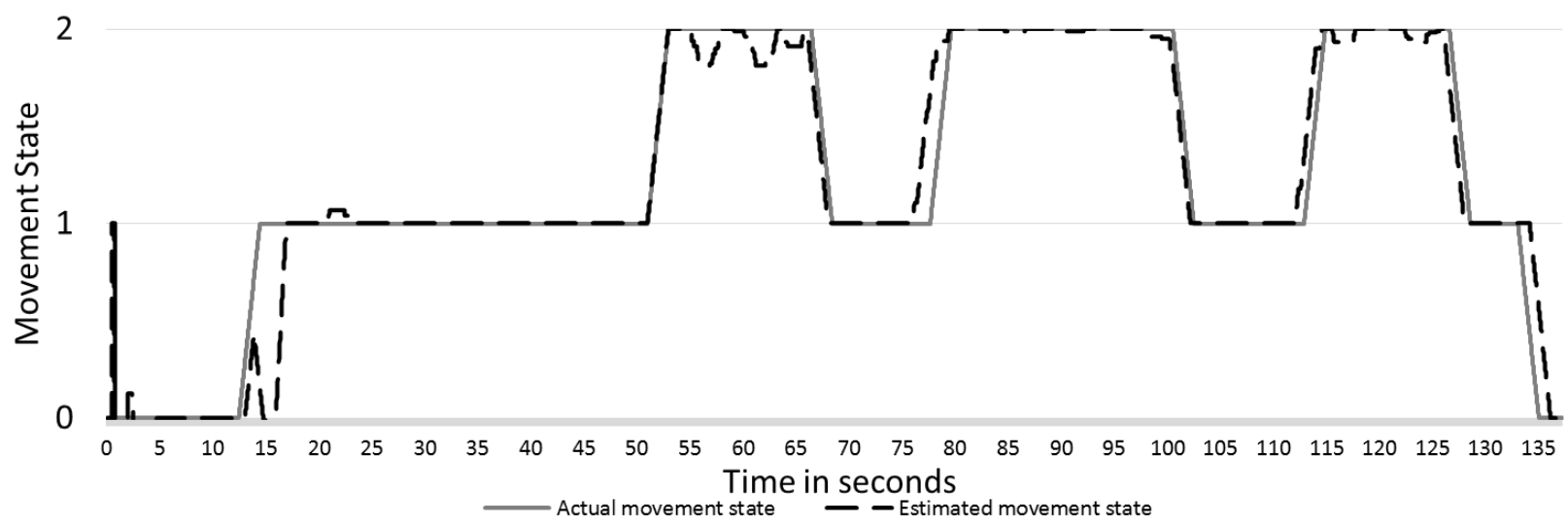

Fig. 8: Example of successful estimation of movement state showing the actual recorded movement state in solid grey and the movement state estimated from the features by the artificial neural network in dotted black

volunteer's speed. While very few samples are exactly correct for the testing run, the black dotted line clearly shows that the system has made a good estimation of the actual speed of the volunteer at nearly every point. Of particular note is the fact that the transitions from one speed to another caused the most uncertainty. This is not unexpected as this is a difficult situation to classify.

It is also interesting to note that the speed of $10 \mathrm{Km} / \mathrm{h}$ has not been estimated well and this may be because less than $2 \%$ of the training was taken at this speed. It is felt that this result would give enough accuracy to help control the prosthetic.

2) Lowest error when estimating movement state: Figure 8 shows the results with the lowest error for the test run when the volunteer's movement state was being estimated where a movement state of 0 represents standing still, 1 represents walking and 2 represents running.

The results have been rounded and averaged and, as can be seen, closely follow the actual movement state. Less than $5 \%$ of the samples are incorrect and the majority of the incorrect samples are around the transition points. As has been previously discussed, this point is open to interpretation as it is possible to walk and run at the same speeds but there is a changeover step where the movement state is half running and half walking as the changeover occurs and this may be exaggerated by the use of the treadmill.

\section{B. Processing time}

The code currently takes approximately 10 seconds to process 10,000 samples recorded at 50 samples a second. This suggests a processing speed of $50 \mathrm{~ms}$ per sample. Classification of the same sample set takes approximately $10 \mathrm{~ms}$. These figures both suggest that real time processing would be possible.

\section{CONCLUSIONS}

In previous work the authors have shown that it was possible to distinguish walking from running using a single EMG signal with an accuracy of over $96 \%$. The new work has incorporated further sensors which have provided two novel and effective features from the outputs of the pressure and accelerometer sensors. While the results are still around $96 \%$ accurate, they now allow the extra state of standing to be identified. They also allow the system to identify the speed at which the volunteer is moving.

It was only possible to achieve a rough approximation of the state and speed of the volunteer using the new features in their raw state. However, after the use of ANN based models both the state and speed could be estimated well. Two Multi-Layer Perceptron based artificial neural networks were trialled. The first implementation used a tansig transfer function. The second used the linear output node response and a scaled conjugate gradient (scg) optimisation method. In 60\% of the tests the second implementation produced a lower error.

The data sets used to create the new features were captured from a volunteer without an amputation. However, data captured from the prosthetic foot and residual limb of a single leg amputee shows identical behaviour. This means that it will be possible to extract the same features from a person using a prosthesis.

As the sensors will be easy to mount and the new features are simple to extract it is likely that this system will work well in real time and thus should support the near real control that will be the focus of further study.

\section{FUTURE WORK}

This work will be developed by working with the data in real time and moving from the treadmill to real running. Further experiments will be carried out using more precise movement criteria so that the changeover points and uncertain areas can be further tested.

\section{ACKNOWLEDGMENT}

The authors would like to thank Ray Foster who volunteered to be tested and Alan McDougall, Senior 
Prosthetist at Chas A Blatchford and Sons Ltd.

\section{REFERENCES}

[1] X. Zhang, Y. Liu, F. Zhang, J. Ren, Y. L. Sun, Q. Yang, and H. Huang, "On design and implementation of neural-machine interface for artificial legs," Industrial Informatics, IEEE Transactions on, vol. 8, no. 2, pp. 418-429, 2012.

[2] P. A. Hardaker, B. N. Passow, and D. Elizondo, "State detection from electromyographic signals towards the control of prosthetic limbs," in Computational Intelligence (UKCI), 2013 13th UK Workshop on, pp. 120-127, IEEE, 2013.

[3] H. Huang, T. A. Kuiken, and R. D. Lipschutz, "A strategy for identifying locomotion modes using surface electromyography," Biomedical Engineering, IEEE Transactions on, vol. 56, no. 1, pp. 65-73, 2009.

[4] P. Robinson, P. Nurse, R. Richter, G. Bugmann, and R. Burns, "Single site myoelectric control of a complex robot hand," in Proceedings of the International Workshop on Advanced Robots and Intelligent Machines, 1997.

[5] R. N. Scott, "Myoelectric control of prostheses and orthoses," Bulletin of prosthetics research, vol. 7, p. 93, 1967.

[6] G. C. Matrone, C. Cipriani, M. C. Carrozza, and G. Magenes, "Real-time myoelectric control of a multi-fingered hand prosthesis using principal components analysis," Journal of neuroengineering and rehabilitation, vol. 9, no. 1, pp. 1-13, 2012.

[7] K. H. Ha, H. A. Varol, and M. Goldfarb, "Volitional control of a prosthetic knee using surface electromyography," Biomedical Engineering, IEEE Transactions on, vol. 58, no. 1, pp. 144-151, 2011.

[8] T. A. Kuiken, L. A. Miller, R. D. Lipschutz, K. A. Stubblefield, and G. A. Dumanian, "Prosthetic command signals following targeted hyperreinnervation nerve transfer surgery," in Engineering in Medicine and Biology Society, 2005. IEEE-EMBS 2005. 27th Annual International Conference of the, pp. 7652-7655, IEEE, 2005.

[9] L. J. Hargrove, A. M. Simon, A. J. Young, R. D. Lipschutz, S. B. Finucane, D. G. Smith, and T. A. Kuiken, "Robotic leg control with emg decoding in an amputee with nerve transfers," New England Journal of Medicine, vol. 369, no. 13, pp. 1237-1242, 2013.

[10] J. Rosen, M. Brand, M. B. Fuchs, and M. Arcan, "A myosignal-based powered exoskeleton system," Systems, Man and Cybernetics, Part A: Systems and Humans, IEEE Transactions on, vol. 31, no. 3, pp. 210 222, 2001.

[11] Z. O. Khokhar, Z. G. Xiao, and C. Menon, "Surface emg pattern recognition for real-time control of a wrist exoskeleton," Biomedical engineering online, vol. 9, no. 1, pp. 1-17, 2010.
[12] L. Carnaz, A. Oliveira, T. Sato, G. Hansson, and H. Coury, "Effects of sensor, trials and knee joint variables on electrogoniometric gait recordings," Brazilian Journal of Physical Therapy, vol. 12, no. 6, pp. 460-467, 2008.

[13] J. Wang, O. A. Kannape, and H. M. Herr, "Proportional emg control of ankle plantar flexion in a powered transtibial prosthesis," in IEEE... International Conference on Rehabilitation Robotics:[proceedings], vol. 2013, pp. 1-5, 2013.

[14] S. G. Karlsson, "User control of lower limb prostheses," 2010.

[15] H. Huang, Y. L. Sun, Q. Yang, F. Zhang, X. Zhang, Y. Liu, J. Ren, and F. Sierra, "Integrating neuromuscular and cyber systems for neural control of artificial legs," in Proceedings of the 1st ACM/IEEE International Conference on Cyber-Physical Systems, pp. 129-138, ACM, 2010.

[16] J. H. Hong and M. S. Mun, "Relationship between socket pressure and emg of two muscles in transfemoral stumps during gait," Prosthetics and orthotics international, vol. 29, no. 1, pp. 59-72, 2005.

[17] E. Scheme, A. Fougner, O. Stavdahl, A. Chan, and K. Englehart, "Examining the adverse effects of limb position on pattern recognition based myoelectric control," in Engineering in Medicine and Biology Society (EMBC), 2010 Annual International Conference of the IEEE, pp. 6337-6340, IEEE, 2010.

[18] P. T. Wang, C. E. King, A. H. Do, and Z. Nenadic, "A durable, low-cost electrogoniometer for dynamic measurement of joint trajectories," Medical engineering $\mathcal{E}$ physics, vol. 33, no. 5, pp. 546-552, 2011.

[19] S. H. Park and S. P. Lee, "Emg pattern recognition based on artificial intelligence techniques," Rehabilitation Engineering, IEEE Transactions on, vol. 6, no. 4, pp. 400-405, 1998.

[20] S. Roberts, P. Nurse, B. R.S., and P. Robinson, "Myoelectric prosthetic upper-limbs: Past and present: a case for further development.," in Medimec Conference, pp. 181-188, September 1995.

[21] G. Cheron, F. Leurs, A. Bengoetxea, J. Draye, M. Destree, and B. Dan, "A dynamic recurrent neural network for multiple muscles electromyographic mapping to elevation angles of the lower limb in human locomotion," Journal of neuroscience methods, vol. 129, no. 2, pp. 95-104, 2003.

[22] V. D. Kalanovic, D. Popovic, and N. T. Skaug, "Feedback error learning neural network for transfemoral prosthesis," Rehabilitation Engineering, IEEE Transactions on, vol. 8, no. 1, pp. 71-80, 2000.

[23] S. Roberts, "An investigation into the control of an upper-limb myoelectric prosthesis," April 2002.

[24] I. Nabney, "Netlab: algorithms for pattern recognition," 2002. 